\title{
Poly(oxanorbornene)-coated CdTe Quantum Dots as Antibacterial Agents
}

Denise N. Williams ${ }^{1}$, Julia Saar ${ }^{2}$, Vera Bleicher ${ }^{2,3}$, Sibylle Rau ${ }^{3}$, Karen Lienkamp ${ }^{2,4}$, Zeev Rosenzweig ${ }^{1}$

'Department of Chemistry and Biochemistry, University of Maryland, Baltimore County, Baltimore, MD

${ }^{2}$ Department of Microsystems Engineering (IMTEK), Albert-Ludwigs-Universittät, Freiburg, Germany

${ }^{3}$ Department of Operative Dentistry and Periodontology, Center for Dental Medicine of the Albert-Ludwigs-Universität, Freiburg, Germany

${ }_{4}^{4}$ Freiburg Institute for Advanced Studies (FRIAS), Albert-Ludwigs-Universität, Freiburg, Germany

\section{Motivation}

The emergence of several multidrug resistant bacterial strains is becoming a global human health challenge. Unfortunately, the development of trug pipeline with treatments ready for clinical use. For this til the scientists are taking new approaches to antibacterial drug design, including through research into antibacterial polymers, lysins, peptides, vaccines, nanoparticles, and the conjugates of several antibacterial entities. This presentation will review research investigating the hypothesis that antimicrobial polymer and antibacterial nanoparticle conjugates could lead to synergistic activity agai

\section{Background}

Butyl poly(oxanorbornene)s (PONs) are a series of polymers which alone have selective, tunable, broad-spectrum activity against bacteria; including against some drug-resistant strains. ${ }^{1}$ Additionally, previous work has shown PONs conjugated to silicon wafers, gold wafers, polymer
matrices, or gold nanoparticles can improve the activity of the polymers matrices, or gold nanop

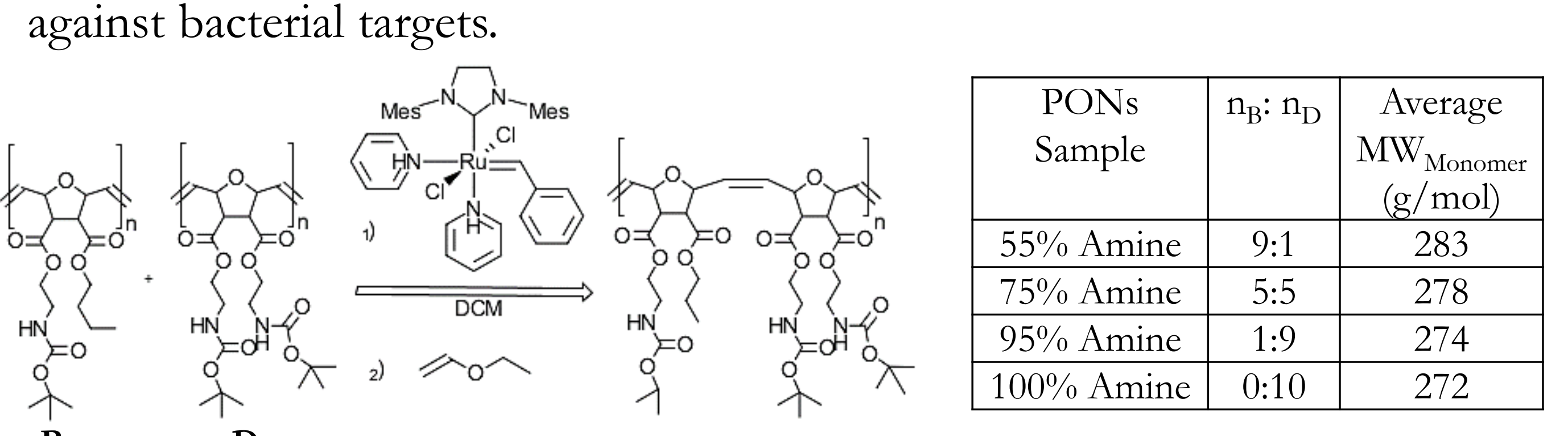

$\underset{\text { Grubbs' third generation catalysts initiated polymerization of }}{\mathrm{D}}$

BOC-protected $\mathbf{B}$ and $\mathbf{D}$ monomers via ring opening metathesis.

The cadmium telluride quantum dot (CdTe QD), with a $2.4 \mathrm{eV}$ bandgap, is a nanomaterial with optimal light-activated antibacterial activity compared to other QD materials and sizes, including against drug resistant

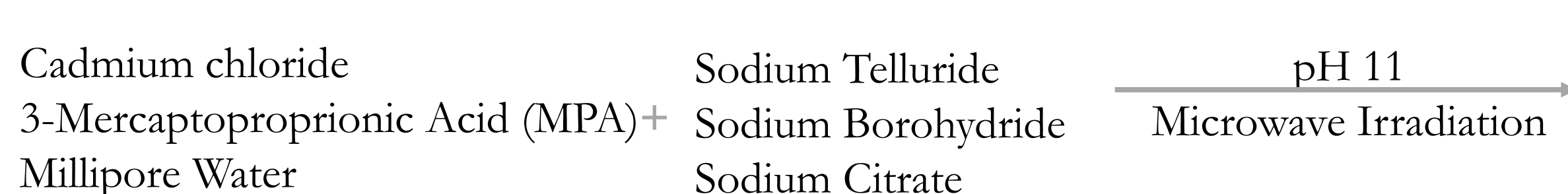

Synthesis scheme for micirowavave synthesis of MPA-CdTe QD.

This project conjugated a series of PONs-CdTe QDs via EDC coupling, and then characterized the conjugation efficiency via zeta potential (A)and thermogravimetric analysis (B) measurements. DCF-DA assays
demonstrated higher ROS generation levels from demontiation, hat that the ROS generation levels of PONs-QDs depend the PONs molecular structure $(\mathrm{C})$.
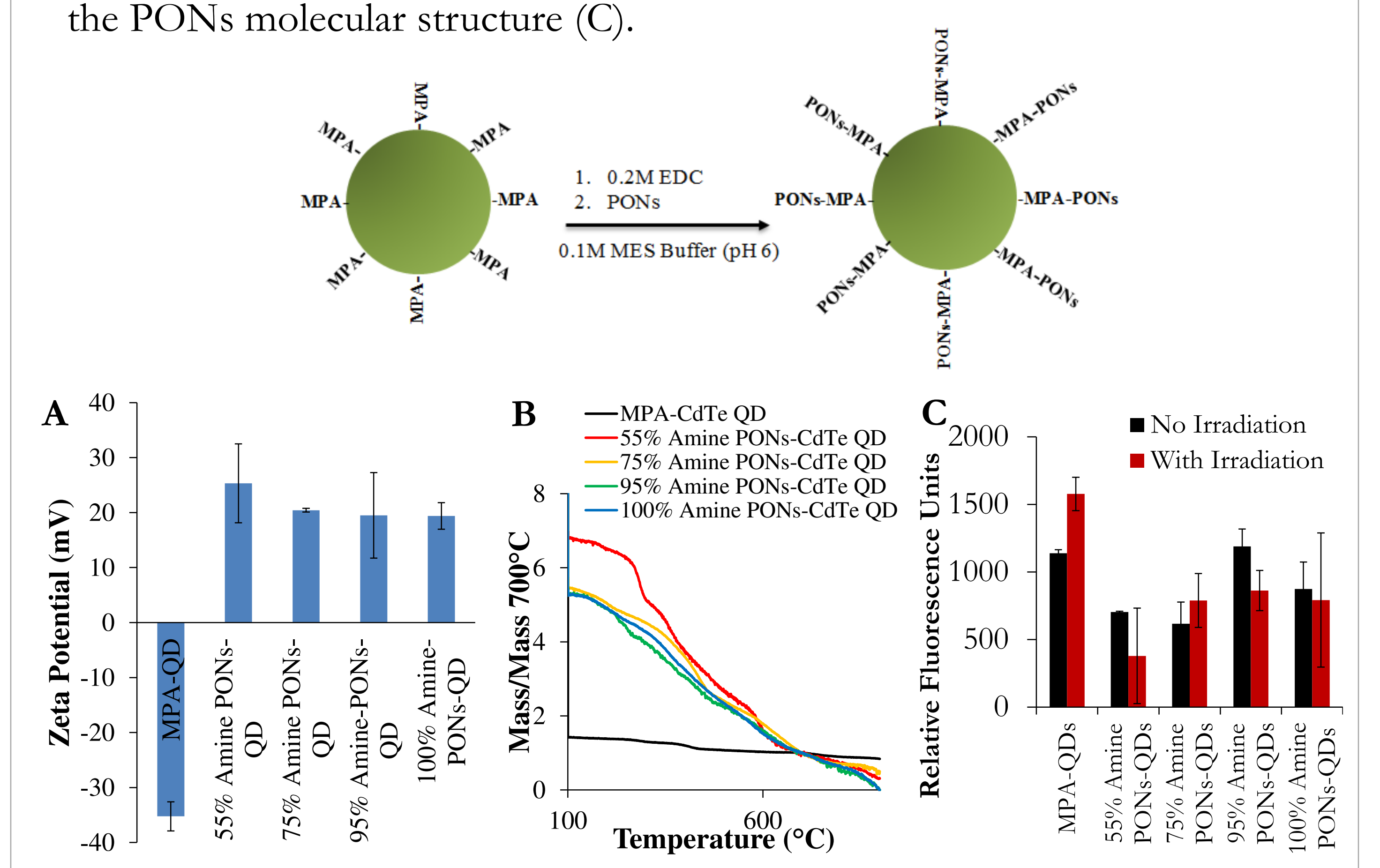

\section{Antibacterial Activity}

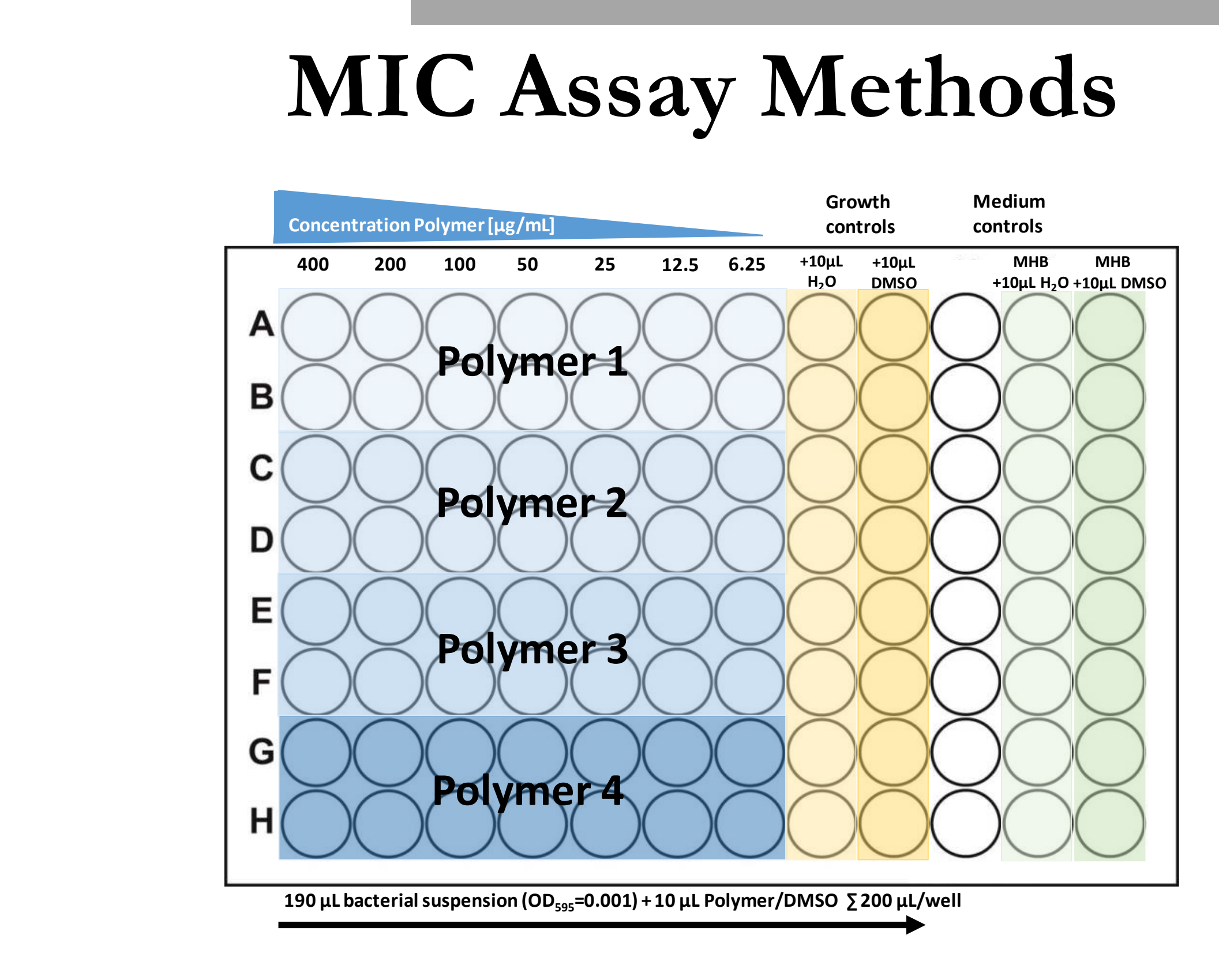

Escherichia coli and Staphylocococus aurreus bacteria were inoculated in fresh Mueller Hinton Broth (MHB) suspensions the day before

Inoculated cell suspensions were washed via centrifugation at fresh MHB at a final $\mathrm{OD}_{555 \mathrm{~mm}}$ of 0.001 .

Cells and teers (PONs, MPA-QD, PONs-QD,

Controls) were put in 96 well plates as illustrated above.

Plates were incubated at $37^{\circ} \mathrm{C}$ overnight.

The following day the $\mathrm{OD}_{505}$ was read and the percent bacteria cell growth was calculated via:

Percent Cell Growth $=\left(\frac{\mathrm{OD}_{\text {Treatment }}-\mathrm{OD}_{\text {Medium control }}}{\mathrm{OD}_{\text {Growht control }}-\mathrm{OD}_{\text {Medium control }}}\right) \times 100 \%$

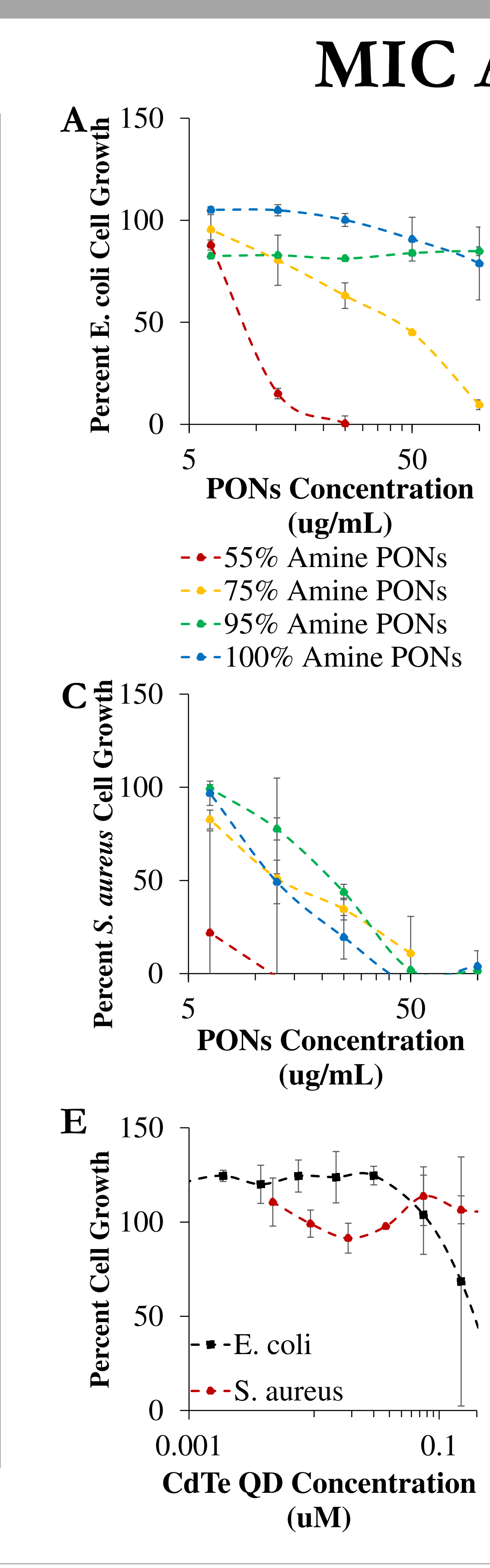

MIC Assay Results

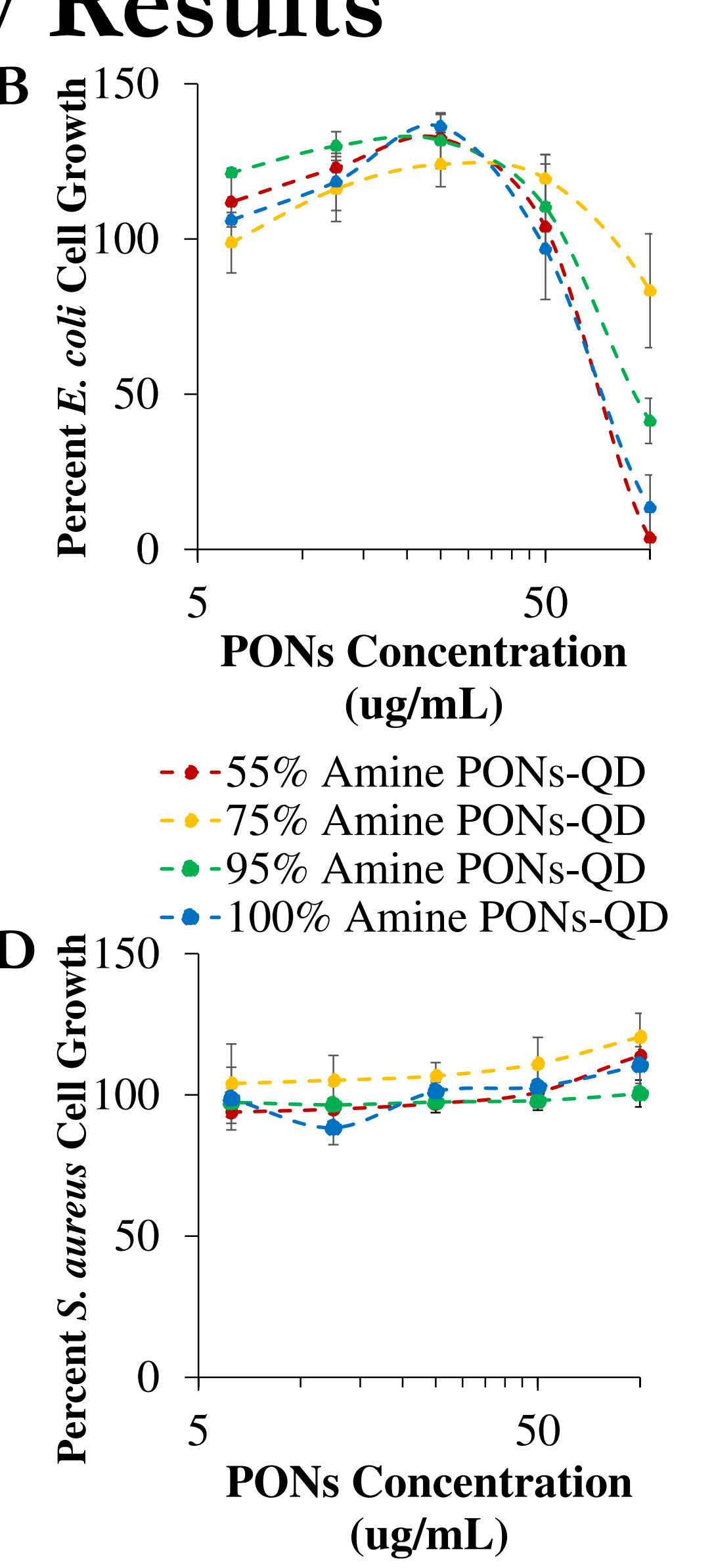

The lower amine content PONs had greater antibacterial activity than the

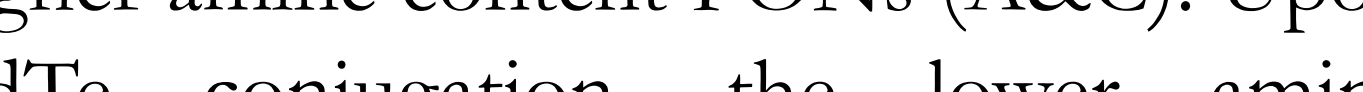
content conjugates had similar activity as higher amine content conjugates against
E. coli (B), but no conjugates werte effective against $S$. aurreus (D). The MPAconcentrations relevant to this study (E).

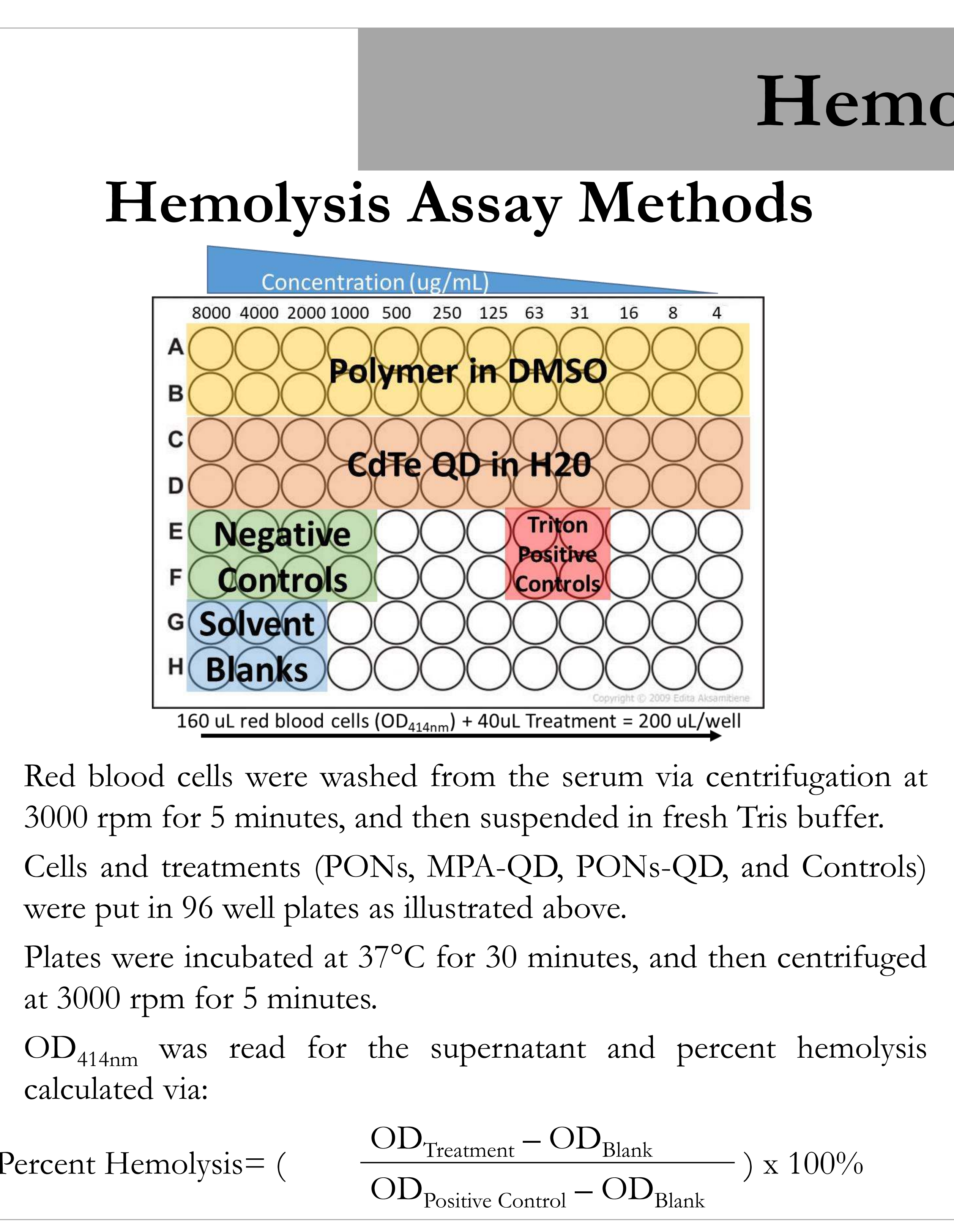

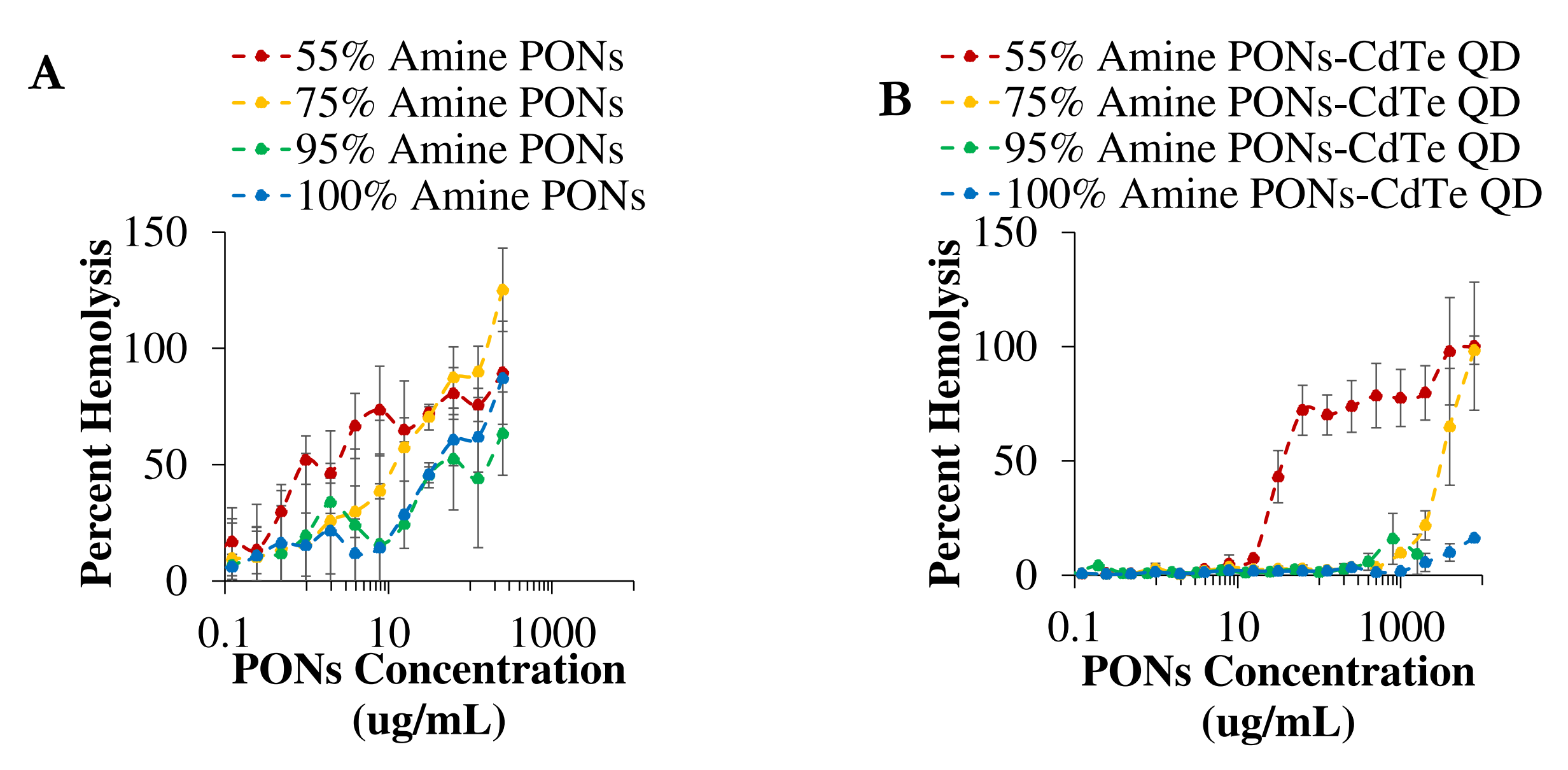

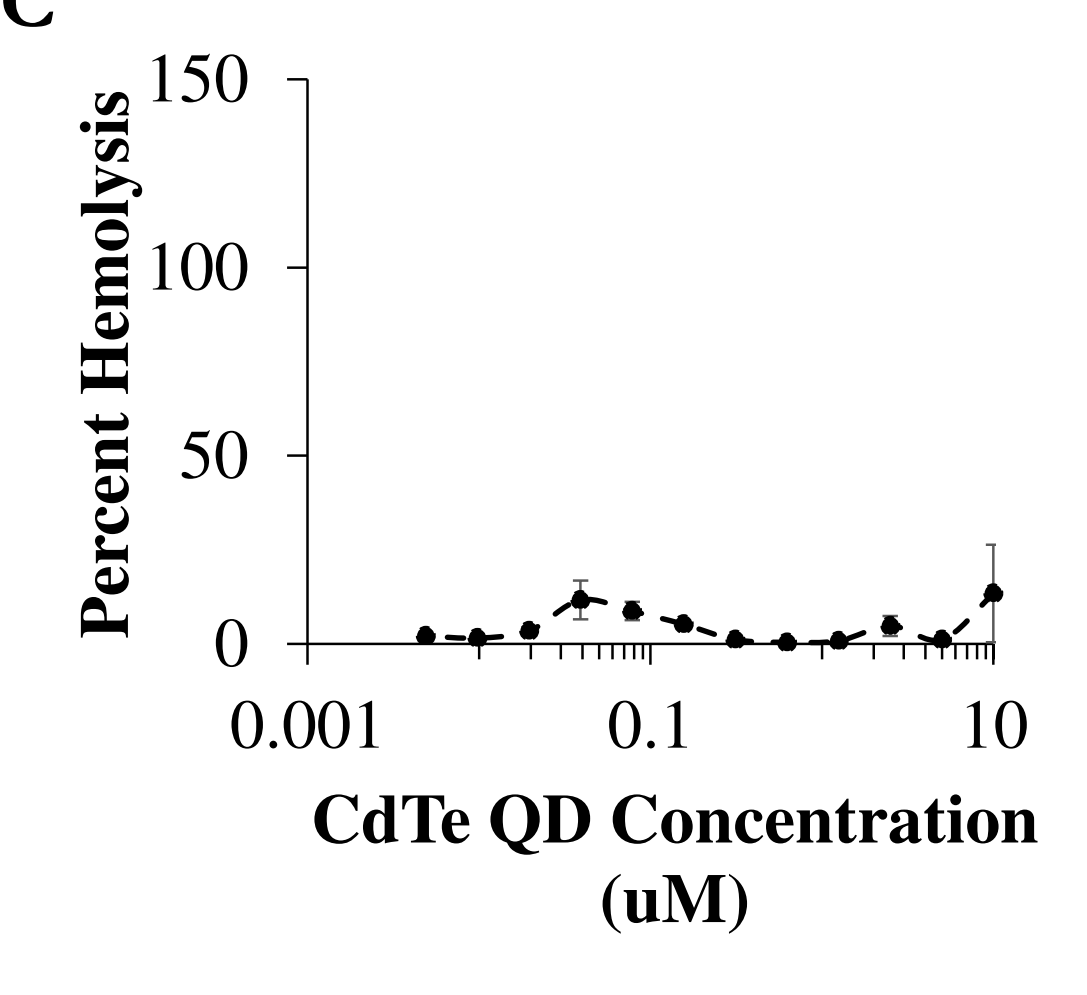

Lower amine content free PONs had greater hemolytic activity than the higher amine content PONs (A). While this hemolytic activity was only evident at higher concentrations of the PONs-QDs Concentrations relevant to this activity a

\section{Therapeutic Index

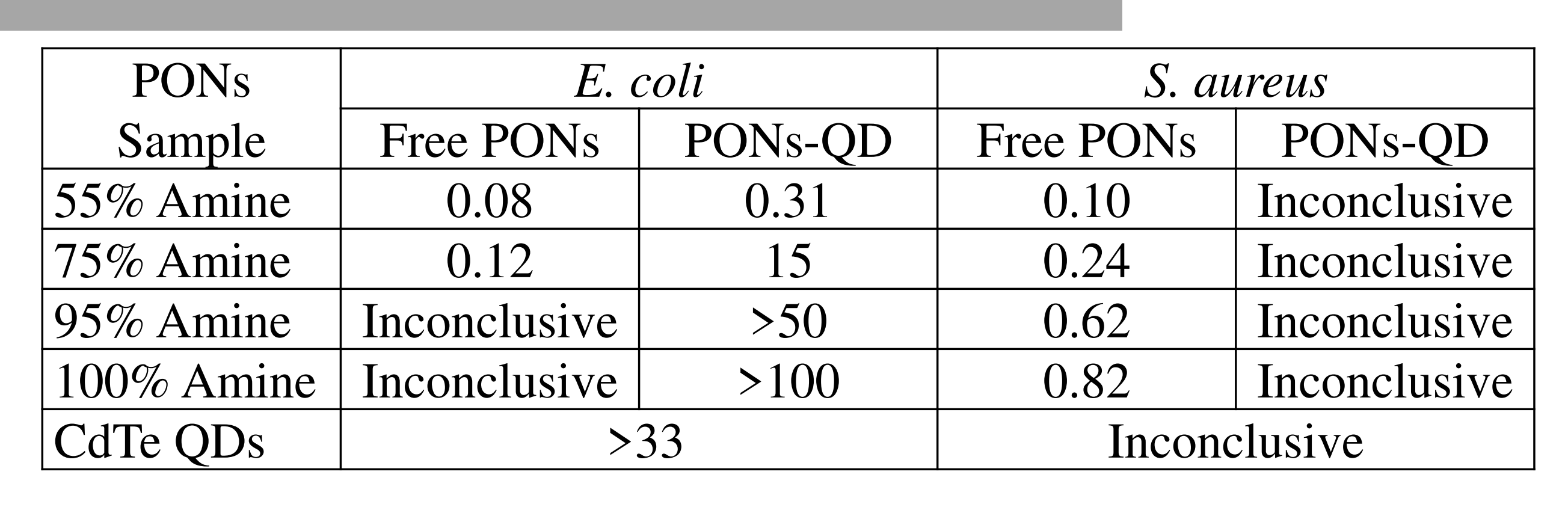 \\ : samples did not have hemolytic activity at tested concentrations}

'Inconclusive': samples did not have antibacterial activity at tested concentrations

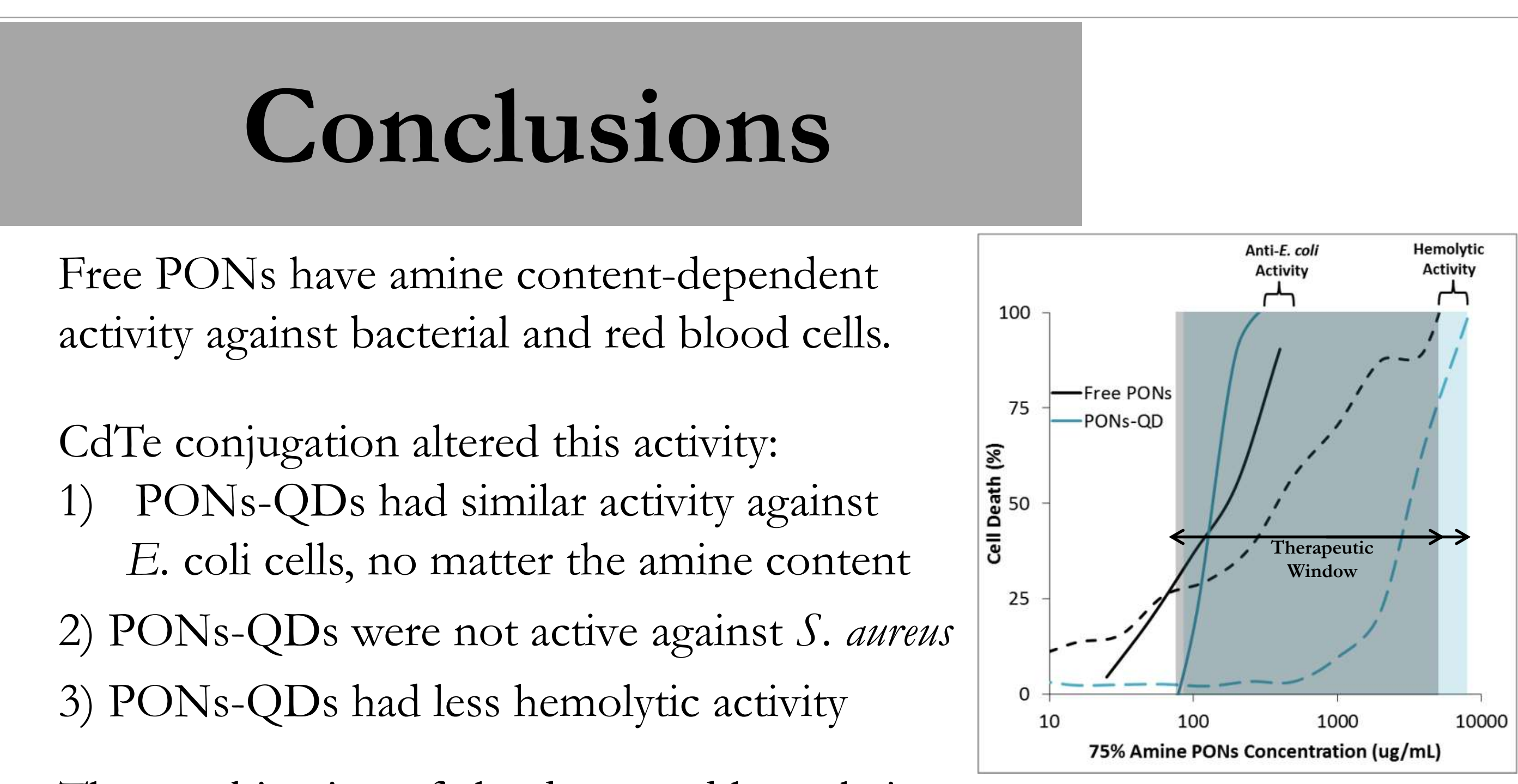
3) $\mathrm{PON}$-QDs had less hemolytic activity

The combination of the decreased hemolytic

activity but maintained anti-E. coli activity lends to wider therapeutic for greater concentrations of PONs to be used in antibacterial treatment while in conjugate form, with less concern for off-target effects.

\section{References}

Study's Publication: Williams, D. N.; Saar, Jj; Bleicher, V.; Rau, S.; Lienkamp, K., Rosenzweig, Z. Poly(oxanorbornene)-coated CdTe Quantum Dots
Antibacterial Agents. ACS Applied Bio Materials. 2020, 3 (2), 1097-1104.

Al-Ahmad, A.; Laird, D.; Zou, P.; Tomakidi, P.; Steinberg, T.; Lienkamp. K., Nature-Inspired, Antinicrobiala Polymers - - Ass
for Biomedical Applications. Plos One 2013,8 8 .

Courtney, C.; Goodman, S.; McDaniel, J.; Madinger, N: Chatteriee, A. Nagpal, P., Photoexcited quantum dots for killing multidrug-resistant bacteria. Nature Materials $2016,15(5), 529$ Wang, K; Li, N;; Hai, X;; Dang, F, Lysozyme-mediated fabrication of well-

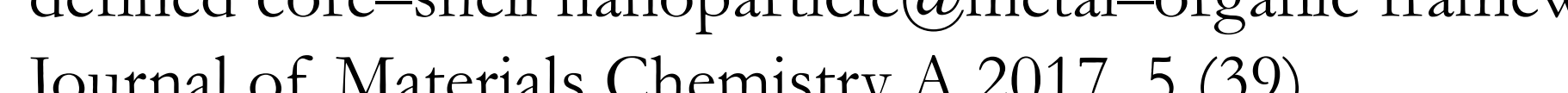
(B). Even directly illuminated, the MPA

\section{Acknowledgements}

This work was primarily supported by National Science Foundation grant supplement award for D.N.W. Work in collaboration with the Lienkamp group was additionally supported
by the National Science Foundation Center for Chemical Innovation (CCD program award for the Center for Sustainable Nanotechnology (CSN)(Grant No. CHE-1503408) Chemistry Biology Interface program under National
training supplement grant number NIH-T32-GM066706.

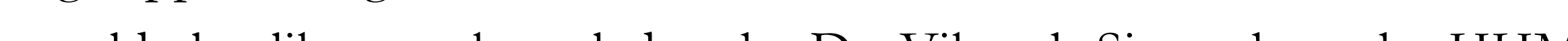
Science Education oroup at UMBC, and UMBC's Molecular Chancterization and Analysis Complex for assistance with is work. A supplemental fellowship support for D.N.W was provided by the UMBC 